\title{
On-Orbit Upset Rate Prediction at Advanced Technology Nodes: a 28 nm FD-SOI Case Study
}

\author{
Victor Malherbe* ${ }^{* \dagger}$, Gilles Gasiot*, Dimitri Soussan*, Jean-Luc Autran ${ }^{\dagger}$, Philippe Roche* \\ *STMicroelectronics, 850 Rue Jean Monnet, F-38926 Crolles Cedex, France. \\ ${ }^{\dagger}$ Aix-Marseille University and CNRS, IM2NP, UMR7334, Marseille, France.
}

\begin{abstract}
We address accurate computation of on-orbit upset rates in advanced technologies, with a focus on FD-SOI at the $28 \mathrm{~nm}$ node. Heavy-ion measurements performed on FD-SOI SRAM bit-cells give experimental evidence of the technology's intrinsic robustness in space environments; this extreme reduction of sensitive volume dimensions deeply affects the assumptions pertaining to the radiation response models used to predict upset rates. The generic "Integral Rectangular ParallelePiped" (IRPP) model, although requiring careful setting of its parameters, provides us with first-order estimates of the error rate. We then present a custom FD-SOI response model within our MonteCarlo simulation chain, enabling comparison with IRPP and further analyses.
\end{abstract}

\section{INTRODUCTION}

Accurate prediction of the rate of Single-Event Upsets (SEU) is of utmost importance for space missions featuring a harsh radiation environment: while under-prediction of the system's SEU rate may result in mission failure, overestimating it can lead to an overly conservative design coming at a high cost, especially given the timescales involved. Because direct measurements of SEU's on orbit are quite rare, the most practical way to enable projections of a circuit's error rate in space is to use a combination of accelerated test data along with space environment models describing the particle spectra encountered on orbit. In many cases, the irradiation experiments can only be performed at normal incidence or just a few angles due to limited beam time; therefore, an extra modeling effort is required to extrapolate the response of the technologies under an omnidirectional space environment.

To that end, several SEU rate prediction methodologies have been developed over the years, for instance the Integral Rectangular ParallelePiped (IRPP) method originally developed in [1] and now implemented in popular tools such as CREME [2] and SPENVIS [3]. However, emerging technologies such as Fully-Depleted SOI (FD-SOI) or FinFET feature strongly-confined geometries that are challenging the existing SEU models [4]. In this study we thus wish to discuss what modeling approaches are relevant in advanced depleted technologies.

This paper is organized as follows: in sections II and III, experimental heavy-ion cross sections are presented for a $28 \mathrm{~nm}$ FD-SOI test vehicle embedding SRAM arrays, and compared with measurements on bulk CMOS counterparts. The next two sections IV and $\mathrm{V}$ discuss how these measurements are used as a basis for extrapolation to real space environments: first-order predictions of the SEU rate are obtained via the IRPP method, and we examine the validity of some crucial aspects in the model. Then, by means of Monte-Carlo simulations tightly calibrated with knowledge of the technology's fabrication process and radiation response, we derive a best estimate for the error rate in our study case. This provides us with generic recommendations regarding the usage of CREME for advanced technologies featuring narrow sensitive volumes such as FD-SOI or FinFET.

\section{TEST CHIP AND EXPERIMENTAL SETUP}

The irradiated test vehicle was designed and manufactured in STMicroelectronics' $28 \mathrm{~nm}$ FD-SOI technology. GDS and real views of the layout are given in Fig. 1: the test chip embeds several flip-flop registers and SRAM cuts, as well as microprocessors with various implementations (reference, radiation hardened...).

The heavy-ion testings were performed at RADEF, Finland [5], in compliance with ESA/SCC basic specification No 25100. Five ion cocktails were used, namely nitrogen, neon, iron, krypton and xenon ions giving rise to Linear Energy Transfers (LET) of $1.83,10.2,18.5,30.4$ and $60.0 \mathrm{MeV} . \mathrm{cm}^{2} / \mathrm{mg}$. Three parts were irradiated with fluences of about $10^{7}$ ions $/ \mathrm{cm}^{2}$ on each run and, except for the Single Event Latchup runs, room temperature conditions were used and the memory arrays' supply voltage was $V_{d d, m e m}=1.0 \mathrm{~V}$.

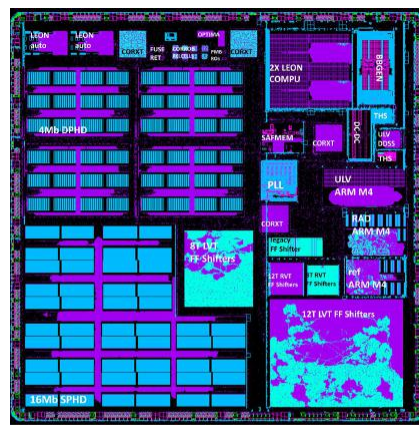

(a)

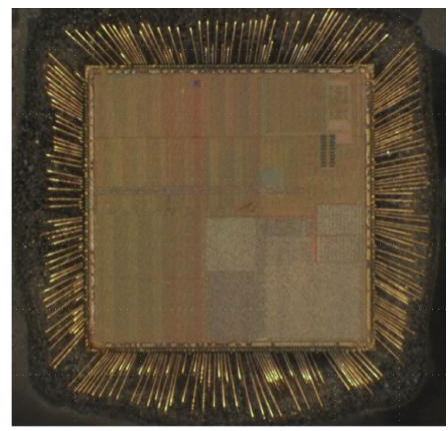

(b)
Fig. 1: Chip layout (a) - photograph after package etching (b). The SRAM cuts occupy the left half of the design.

\section{HeAVy-ION TEST RESUlts}

The overall outcome of this test campaign is a very high intrinsic resilience exhibited by the FD-SOI technology under 
heavy ions. Previous publications had already reported the outstanding SEU hardness of $28 \mathrm{~nm}$ FD-SOI under terrestrial particles such as alphas and neutrons [6]-[7]; this work confirms the technology's hardness in spatial environments as well, with heavy-ion cross sections routinely achieving 2 decades of reduction when compared to equivalent-node bulk CMOS technologies. As far as SEU's are concerned, this extreme resilience of FD-SOI is primarily due to two factors: first of all the very small volume for ion-induced charge deposition, thanks to the $7 \mathrm{~nm}$-thin active silicon film being enclosed by the Buried Oxide (BOX). Second of all, parasitic bipolar amplification is quite limited in this technology, as was investigated with TCAD simulations in [8], leading to collected charges as small as 0.1 to only a few fC.

To help narrow down our discussion, the rest of this study will focus on just one of the SRAM cells tested. The bit-cell follows a classical design with six transistors (Fig. 2), out of which three are SEU-sensitive depending on whether a 0 or a 1 is stored. For instance, in retention mode the bit- and wordlines are not loaded $(B L T=B L F=W L L=W L R=0)$ and if the cell is at $1(B L T I=\overline{B L F I}=1)$, then only PD1 (pull-down NMOS), PG1 (pass-gate NMOS) and PU2 (pullup PMOS) will host an electric field sufficient to separate the radiation-induced electron-hole pairs.

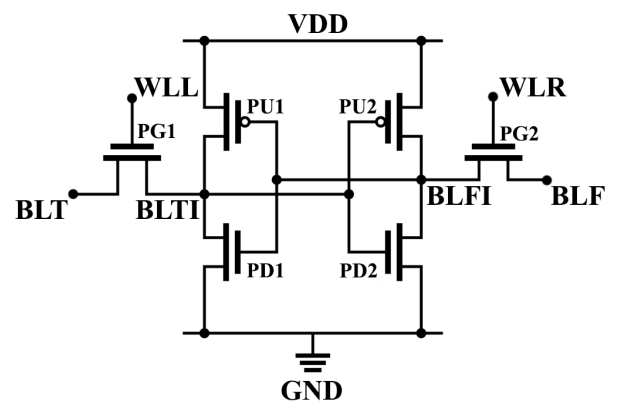

(a)
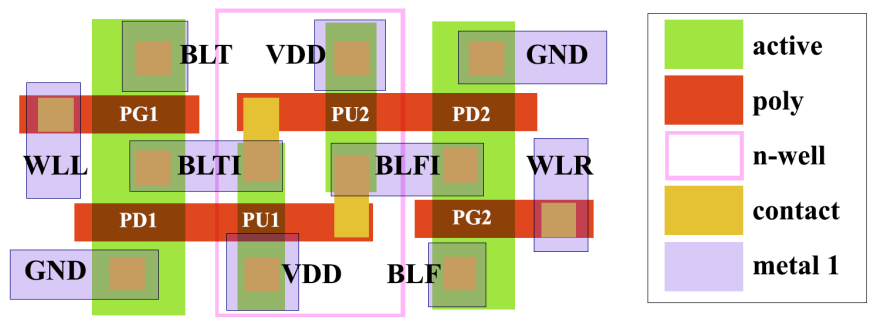

(b)

Fig. 2: The studied 6-transistor SRAM bit-cell:

(a) its netlist and (b) its - idealized - layout

The experimental heavy-ion cross sections collected in the beam tests for our FD-SOI bit-cell are shown in Fig. 3 along with a Weibull fit. As an element of comparison, the cross section for a $28 \mathrm{~nm}$ "bulk-equivalent" bit-cell was also plotted (identical area and similar design, give or take some Design Rule Check alterations). As can be seen, the FD-SOI cell is about two decades less sensitive than the bulk one: the bulk bitcell exhibits an asymptotic cross-section of about 20 times the cell area, meaning that on average each impact triggers a 20- cell upset, because the carriers are free to diffuse on very long ranges. On the other hand, the FD-SOI limiting cross section $\sigma_{\text {sat }}$ is only about $15 \%$ of the cell area thanks to the full dielectric isolation provided by the BOX. In bulk technology, the primary sensitive area is that of the drains of blocked transistors (with a preponderant vertical field) and it only requires an LET of about $1.5 \mathrm{MeV} . \mathrm{cm}^{2} / \mathrm{mg}$ to be "activated". However in FD-SOI the situation is very different: the sensitive area is rather located by the off-transistor channels featuring a strong lateral field - and it takes an LET of about $15 \mathrm{MeV} . \mathrm{cm}^{2} / \mathrm{mg}$ to "activate" it entirely. Such considerations will be used to construct the sensitive volumes for charge collection in the next sections where error rates are derived.

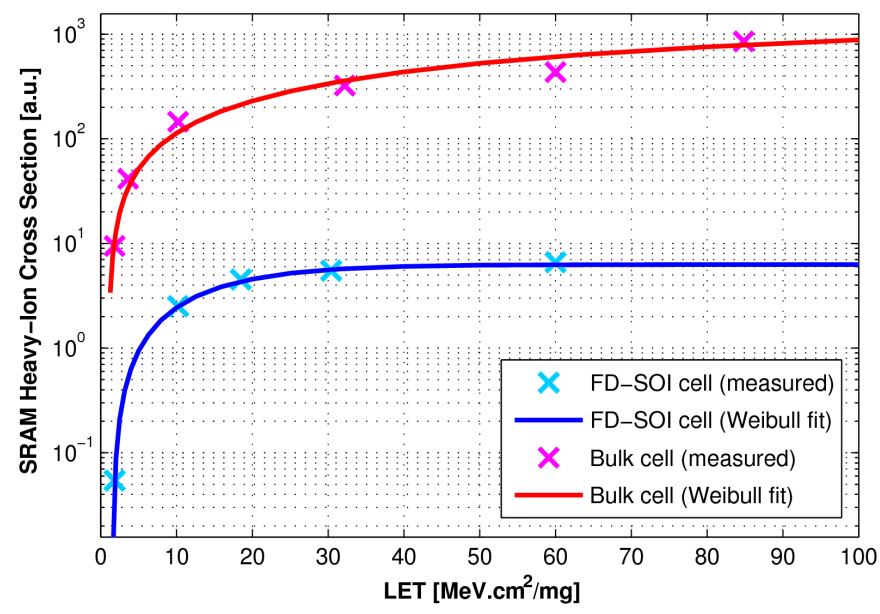

Fig. 3: Experimental heavy-ion cross sections of the bit-cells, exhibiting FD-SOI's intrinsic robustness compared to bulk

\section{First-order SEU RATE ESTIMATE With CREME TOOL SUITE}

The aim of this section is to describe the flow followed to compute on-orbit SEU rates in FD-SOI using CREME tools provided online [2]-[9]. We first simulate ion environments encountered under various orbit and solar conditions, and then make use of the IRPP method to convolve the environment with the experimental test data. We also discuss the influence of crucial parameters in the IRPP setup.

\section{A. Radiation environment simulation}

Several radiation environments were simulated in CREME, ranging from Low Earth Orbit (LEO) to Geosynchronous Earth Orbit (GEO). Trapped protons are accounted for with the AP8 model, while Galactic Cosmic Rays (GCR) are calculated with CREME96 GCR model. We cover multiple solar conditions, namely minimum and maximum "solar quiet" configurations (respectively maximizing and minimizing GCR fluxes) and "worst week" solar flare. All ion species up to Z=92 (uranium) are considered for flux calculations, and transported through 100 mils of aluminium. A sample of resulting LET spectra is plotted in Fig. 4, highlighting differences not only in total flux magnitude, but also in LET content: the GEO environment under quiet Sun contains heavier species, while the LEO 
or flare environments are dominated by light particles. Note that similar fluxes were obtained with SPENVIS and are not reported: the scope of this study is not to discuss the accuracy of radiation environment models, but rather on the SEU models taking those fluxes as an input.

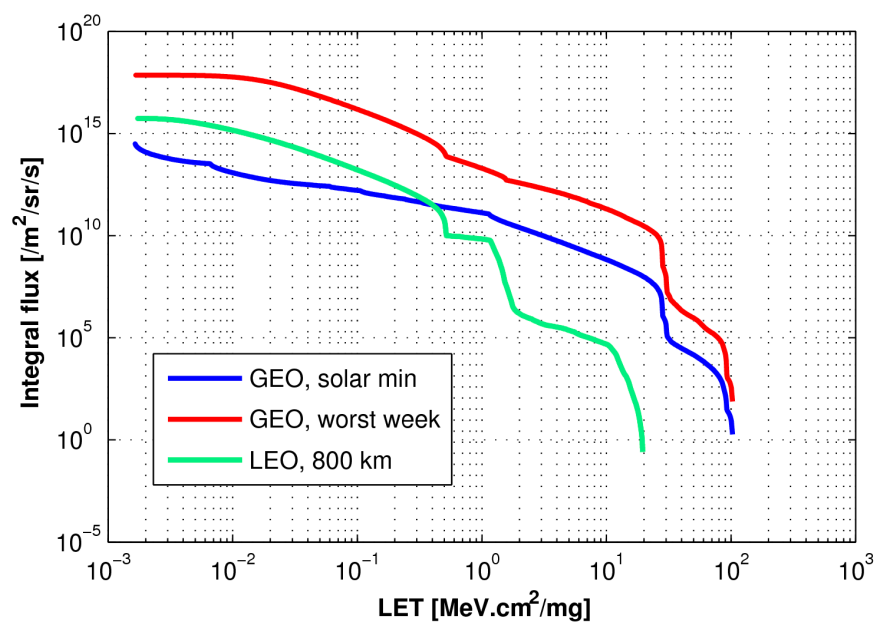

Fig. 4: Integral LET spectra simulated with CREME in various environments: GEO under quiet Sun features high-LET species, while LEO or active Sun environments are dominated by low-LET protons and alphas

\section{B. IRPP method and discussion}

The IRPP method [1], due to its genericity and large case coverage, has been widely adopted in the radiation effects community to compute SEU rates in space from groundbased test data. First of all, given a fixed critical charge $Q_{\text {crit }}$, the (non integral) RPP method works by considering a sensitive volume of $x, y, z$ dimensions chosen to match both the experimental saturation cross section at normal incidence: $x \cdot y=\sigma_{\text {sat }}$ and threshold LET: $L E T_{t h} \cdot z=Q_{\text {crit }}$. Then the angular response is extrapolated by assuming that the sensitive volume has uniform $100 \%$ collection efficacy: for an ion of given LET and direction, this means that the collected charge will equal the charge deposited within the sensitive volume, yielding $Q_{\text {coll }}=Q_{\text {dep }}=L E T \cdot l$ where $l$ is the chord length traveled by the ion within the sensitive volume. This collected charge gives rise to an upset when it is superior to $Q_{\text {crit }}$. Thus, in the RPP method, the predicted cross section at normal incidence is necessarily a step function. The more general IRPP method takes the above ideas further, by interpreting the $\sigma(L E T) / \sigma_{\text {sat }}$ relative cross-section curve as a cumulated distribution function for the threshold LET's of RPP's of identical dimensions: while a step-function cross section curve corresponds to a single threshold LET, a gradual Weibull curve starting with an onset LET represents a wider distribution of threshold LET's from the onset LET value onwards. Note however that the physical meaning of a statistical superposition of identical sensitive volumes with different threshold LET's is quite questionable: at the time in 1992, it was believed that each individual bit-cell would exhibit a sharp cross-section curve, and that the smooth curve measured was due to a spread across the memory array due to process variability. We now

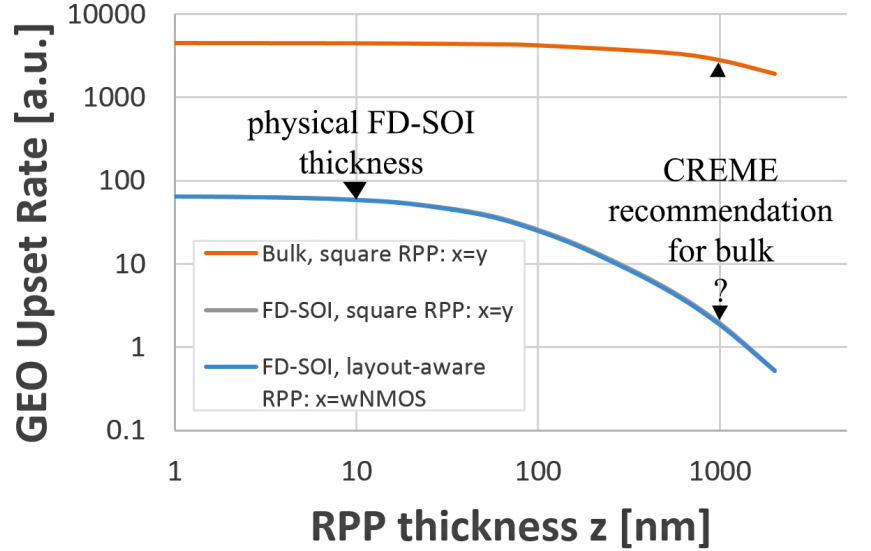

(a)

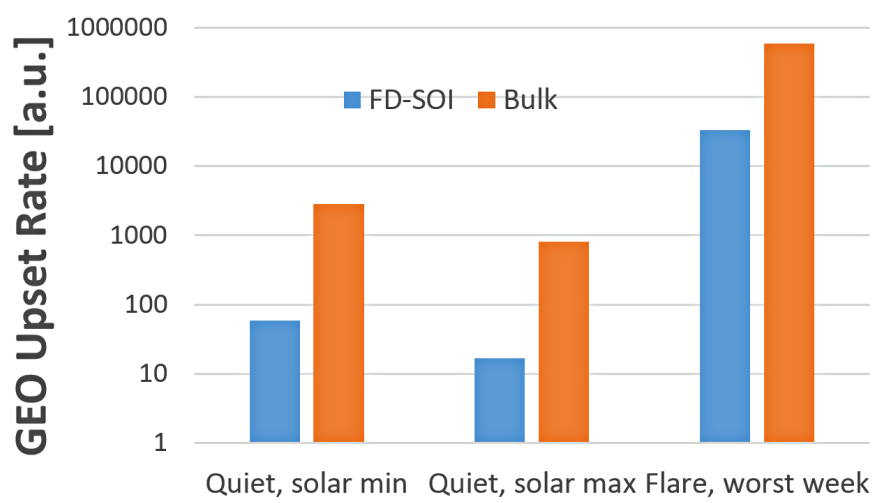

Solar Conditions

(b)

Fig. 5: GEO upset rates obtained with CREME via the IRPP model for FD-SOI and bulk technologies (a) for various RPP dimensions and (b) for various solar activities

know that even one cell alone intrinsically exhibits a gradual activation, which is the motivation for using "nested" sensitive volumes in certain Monte-Carlo codes such as MRED [10].

Given an experimental cross section curve, the $x$ and $y$ dimensions of the IRPP can reasonably be estimated, starting from $\sqrt{\sigma_{s a t}}$ (the default setting in CREME) and then playing with various aspect ratios. However, the choice of the $z$ dimension can be quite blurry; physically, it represents the depth over which quick collection by electric drift occurs, i.e. the depletion depth. In bulk CMOS technologies, the usual recommendation in CREME is to set $z$ around $1 \mu \mathrm{m}$. This is unrealistic for our $28 \mathrm{~nm}$ FD-SOI technology here, whose active silicon thickness is $7 \mathrm{~nm}$ (charges deposited in the BOX or below are not collected). SPICE simulations were performed on the studied bit-cell and revealed critical charges $Q_{c r i t}$ of about $0.4 \mathrm{fC}$ (resp. $0.8 \mathrm{fC}$ ) for NMOS (resp. PMOS) impacts. Together with $L E T_{t h}=1.5 \mathrm{MeV} \cdot \mathrm{cm}^{2} / \mathrm{mg} \Longleftrightarrow 15.45 \mathrm{fC} / \mu \mathrm{m}$, this suggests an effective collection depth of a few dozen nanometers, which is already a lot more in line with the physical sensitive volume. Fig. 5 (a) depicts the $z$ dependence of the SEU rate predicted in GEO for the FD-SOI and bulk bit-cells with the IRPP method, using the Weibull fits from 


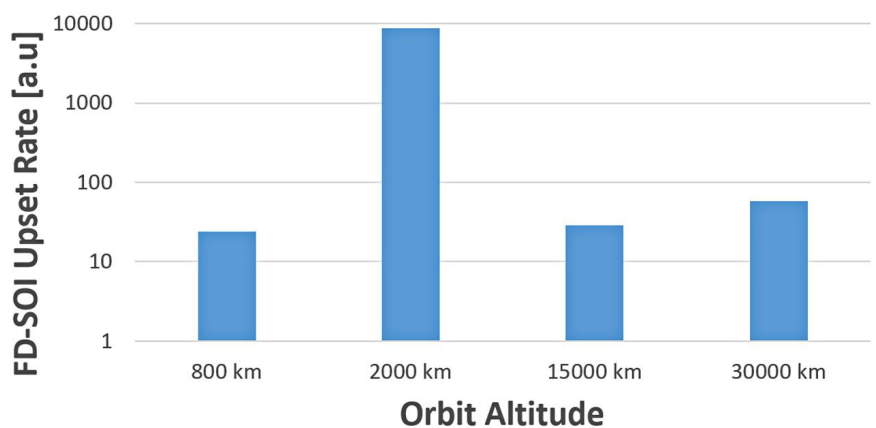

(a)

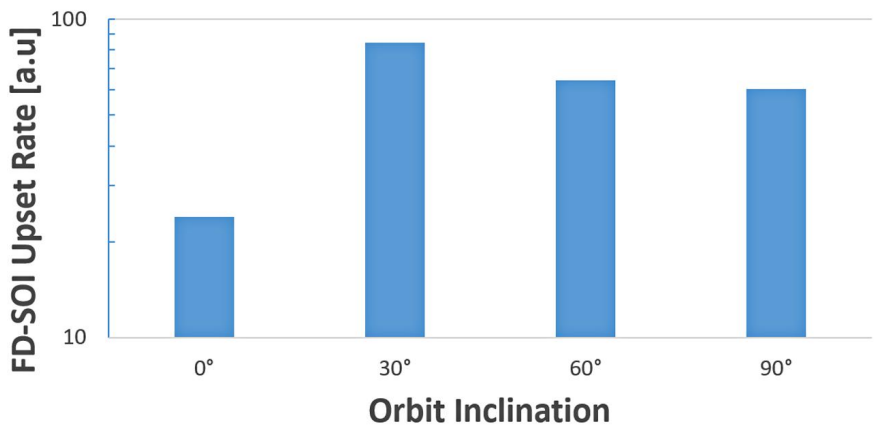

(b)

Fig. 6: Upset rates obtained with CREME via the IRPP model for FD-SOI (a) for various altitudes at 0 inclination and (b) for various inclinations at $800 \mathrm{~km}$

Fig. 3. While the bulk rate - much larger than FD-SOI's - is relatively stable as $z$ varies around its recommended value, the FD-SOI rate drops by a $30 \times$ factor from plausible $z$ values to the $z$ values recommended for bulk technologies. This suggests that very careful analysis of the IRPP outputs is necessary at advanced technology nodes with strong confinement at play. Note that the increase in SEU rate with decreasing $z$, although counter-intuitive at first ("how can a smaller volume be more sensitive?"), is easily understood either in geometrical terms (few chords longer than $z$ for a "needle", and the opposite for a "slab") or in electrical terms (low $Q_{\text {crit }}$ for a small $z$ ). The IRPP $x: y$ aspect ratio was also investigated using information from the cell layout: since the NMOS critical charge is much lower than for the PMOS and since they have much larger area, the sensitive volume $x$ dimension can reasonably be approximated by the NMOS width. However, as shown in Fig. 5 (a), this has very little effect on the SEU rate prediction, giving no more than $5 \%$ correction from the "square IRPP" predictions - and the difference becomes negligible as $z$ gets much smaller than $x$ and $y$, i.e. in the "slab" configuration where chord lengths are driven by $z$.

Using $z=10 \mathrm{~nm}$ for FD-SOI and $z=1 \mu \mathrm{m}$ for bulk, in Fig.5 (b) we compare both technologies' upset rates for various conditions. As can be seen, it takes a "worst week" solar flare for FD-SOI to suffer more upsets than bulk in quiet solar conditions. In Fig. 6 we also study the influence of orbit parameters: for the LEO's, the sharp difference between $800 \mathrm{~km}$ and $2000 \mathrm{~km}$ reflects the presence of the first Van Allen belt, with higher trapped proton populations above approximately $1000 \mathrm{~km}$. The more modest increase in upset rate from $15000 \mathrm{~km}$ to $30000 \mathrm{~km}$ is not due to trapped particles (since the second Van Allen belt is populated with electrons that are mostly stopped by 100 mils of shielding), but rather by the depletion of the Earth's magnetic shield towards higher altitudes. Finally, at altitudes as low as $800 \mathrm{~km}$ the orbit inclination can have a strong impact, especially in the South Atlantic Anomaly.

Note that throughout this study we only consider direct ionization by the ion species on orbit, thereby neglecting upsets from secondary nuclear products: we do account for the trapped protons' contribution to the received LET spectrum, but overlook the upsets triggered by ionizing products of protons against silicon. At LEO altitudes ( $2000 \mathrm{~km}$ and below) such a choice might not be justified, but it allows comparison on a common ground for the various models under discussion.

\section{Best estimate of THE SEU RATE IN TIARA}

In this section we go through the derivation of a more accurate SEU rate by means of a dedicated response model for FD-SOI. We compare the results obtained with those from CREME and give insights into their physical meaning.

\section{A. Overview of TIARA flow}

TIARA stands for ToolsuIte for rAdiation Reliability Assessment, and is a proprietary tool developed at STMicroelectronics to study Single Event Effects (SEE) at circuit level [11]. TIARA's flow for prediction of SEE's can be summarized followingly:

- Structure generation from circuit netlist and layout: using the $\mathrm{x}$ and $\mathrm{y}$ coordinates from the GDS together with process information ("the $\mathrm{z}$ coordinates" such as implantation depths, Back-End-Of-Line or BEOL dielectric thicknesses), a 3D structure is generated for the circuit. Fig. 7 depicts the 3D structure generated for the bit-cell in this study, up to the third metal layer (same coloring as Fig. 2 (b)). Then considering the circuit netlist, a Layout-Versus-Schematic (LVS) pass is performed to automatically map layout locations to nodes/devices in the schematic.

- Particle ray-tracing for charge deposition: a virtual irradiator is defined to reproduce a particle environment via Monte-Carlo sampling. Particles are randomly drawn and then traced in the circuit structure, both geometrically (ray-triangle intersections for material boundary traversals) and physically (energy loss along particle track by direct ionization using SRIM stopping power tables), giving rise to charge deposition. For each particle, this outputs a distribution of radiation-induced carriers in the structure.

- Charge collection and circuit response: TCAD-calibrated fast physical models are called to relate the deposited carriers to collected current waveforms, accounting for (slow) diffusion processes and (fast) electric drift. At this point, the LVS link dictates what netlist node to inject the currents at, depending on physical location of the charge collection. The circuit's response to these currents 
is finally computed in the SPICE solver, allowing the calculation of aggregates such as cross sections or SEU rates typically, or custom analysis if needed.

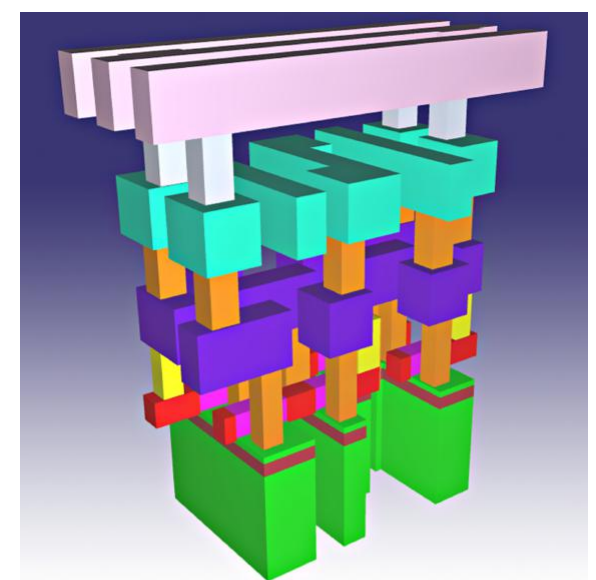

(a)

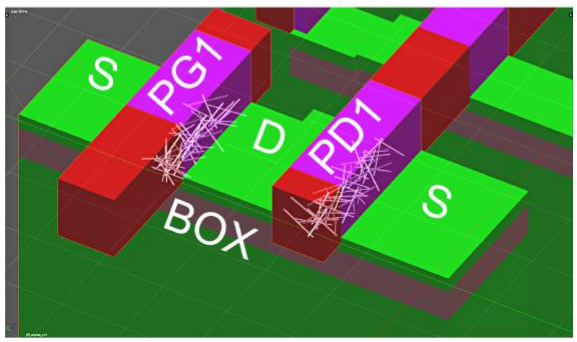

(b)

Fig. 7: TIARA 3D structure for FD-SOI bit-cell simulation: (a) Global view up to the power rails (not displayed for clarity sake: all dielectrics except the BOX, substrate underneath shallow trench isolation)

(b) Close-up on alpha particle tracks triggering SEU's within sensitive NMOS channels

\section{B. FD-SOI radiation response model and calibration at nor- mal incidence}

The main specificities of our $28 \mathrm{~nm}$ FD-SOI technology under radiations have been investigated with TCAD simulations and were reported in [8]. The primary parameter to account for, beside the sensitive volume small dimensions, is the bipolar amplification defined as the ratio of the collected charge to the deposited charge. In an NMOS for instance, radiation-induced holes in the body region tend to lower the potential barrier and trigger the parasitic lateral bipolar transistor, giving rise to current injection by the source. This phenomenon is intrinsic to all SOI technologies where majority carriers can linger in the body region. In $28 \mathrm{~nm}$ FD-SOI, the $\beta$ factor was shown to be no more than 4 for LET's above $1 \mathrm{MeV} . \mathrm{cm}^{2} / \mathrm{mg}$.

Expanding on the work done in [8] where a constant bipolar amplification was accounted for in TIARA, we devise our response model for FD-SOI with a spatially-varying collection efficiency $\eta$ to modulate the LET-dependent bipolar amplification along the gate axis of each sensitive transistor. To capture the non-uniformities of this charge collection "yield", the ion track inside the active silicon is discretized at points $y_{i}$ (with nanometric resolution typically). Then, the total collected charge is computed as:

$$
Q_{\text {coll }}=\sum_{i} Q_{\text {dep }}\left(y_{i}\right) \cdot \beta(L E T) \cdot \eta\left(y_{i}\right)
$$

Using this method, for any impact we are able to calculate the collected charge $Q_{\text {coll }}$. It is then related to a doubleexponential current waveform of rise and fall times $\tau_{r}$ and $\tau_{f}$ by:

$$
i(t)=\frac{Q_{\text {coll }}}{\tau_{f}-\tau_{r}} \cdot\left[\exp \left(-t / \tau_{f}\right)-\exp \left(-t / \tau_{r}\right)\right]
$$

and this is what ultimately feeds the SPICE solver.

As a summary, the "free" parameters in our response model are the LET-dependent bipolar amplification, the positiondependent yield, and the current waveform rise and fall times. To fix all of these, we use TCAD simulations to provide us with orders of magnitude and relative trends, while still relying on calibration against heavy-ion data at normal incidence to set the precise figures:

- $\beta(L E T)$ relative trends for NMOS and PMOS were obtained in TCAD, and precise numbers are obtained to match the observed threshold LET (Fig. 8 (a)).

- $\eta(x)$ is a non-uniform weight between 0 and 1 along the gate axis (Fig. 8 (b)). In accordance with works such as [12], it is set at 1 at the drain-channel junction and goes to 0 further away. Note that although the drain is strongly doped (while the channel is intrinsic), charge collection does not vanish immediately inside it. Defining the spatial dependence of this yield truly requires nanometric resolution around the junction, for the experimental heavyion cross section to be matched. (Fig. 8 (c)). Note that for other gate lengths than $L_{m i n}$, the function is simply stretched by the $L_{g} / L_{\min }$ ratio. This is confirmed by our TCAD simulations, showing only small variations in collection for other transistor lengths.

- $\tau_{r}$ and $\tau_{f}$ the rise and fall times of the double-exponential waveforms are both below $10 \mathrm{ps,} \mathrm{representative} \mathrm{of} \mathrm{the}$ current shapes computed in TCAD; since the critical charge, roughly speaking, scales as $C . V+I . \tau$, modulating such small values of rise and fall times with the LET or the position would make little difference to the end result.

- Finally, further simulations suggest that the supply voltage $V_{d d}$ only has a weak influence on all of the above parameters (e.g. $10 \%$ decrease in $Q_{\text {coll }}$ for $-50 \% V_{d d}$ ): while in bulk technology, the depletion depth strongly expands with $V_{d d}$, resulting in deeper collection, in depleted technologies like FD-SOI the collected charge is primarily dictated by the - rigid - sensitive volume.

Using those settings for our parameters, we reproduce the heavy-ion measurements with an excellent agreement. We then perform some sanity checks to ensure that the angular response is properly captured. Namely, simulations of alpha Soft Error Rate (SER) were performed and confronted to experimental test results across a variety of bit-cells and supply voltages. The $\alpha$-SER was predicted with $1.5 \times$ accuracy under 


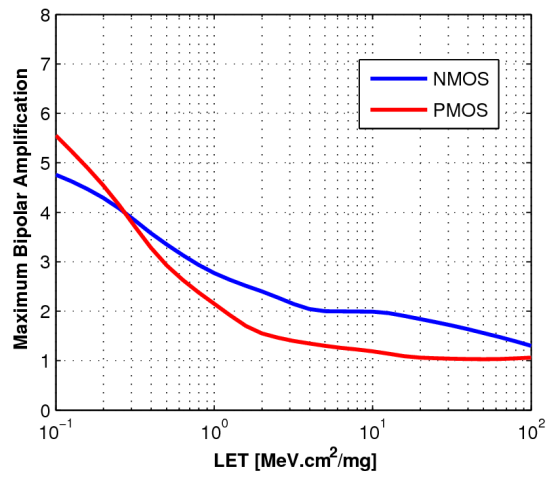

(a)

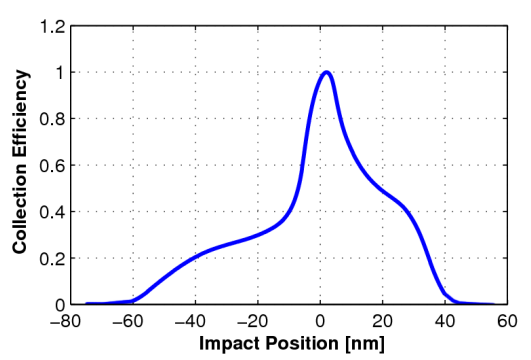

(b)

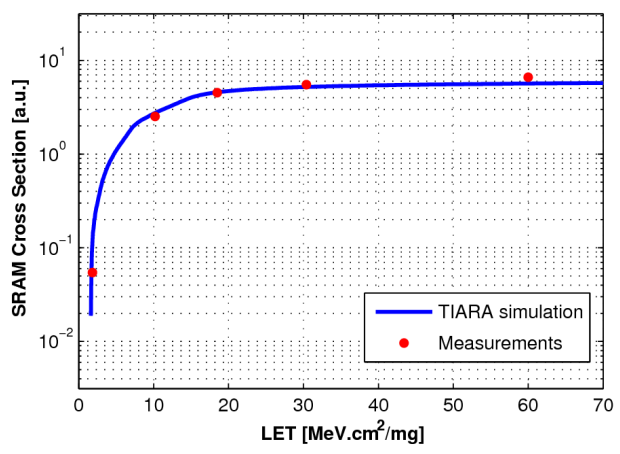

(c)

Fig. 8: Breakdown of TIARA's dedicated response model for FD-SOI: (a) LET-dependent bipolar amplification - (b) Nonuniform collection efficiency - (c) Simulated heavy-ion cross section after calibration phase

all these test conditions, giving us confidence that not only the geometrical effects (probed with the isotropic environment provided by the alpha source) are rightfully accounted for, but also that electrical properties are well handled by the simulator.

\section{TIARA SEU rate calculations in FD-SOI}

Once the calibration phase is performed, the response model in TIARA can be used to predict upset rates in arbitrary environments. Provided an isotropic integral LET spectrum given by CREME $(\phi(L E T)$ in particles per unit time, area and steradian, with total flux $\phi(0))$, the most direct way of calculating the upset rate is to consider the normalized flux $\phi(L E T) / \phi(0)$ to be a probability distribution for drawing LET's randomly in the Monte-Carlo simulator. This reproduces the natural distribution in the environment (alternatively, importance sampling could be implemented but is not discussed here). Under those conditions, the upset rate $R$ in events per unit time is simply given by:

$$
R=4 \pi \cdot \phi(0) \cdot \frac{A_{\text {irrad }}}{4} \cdot \frac{N_{S E U}}{N_{\text {impacts }}}
$$

where $N_{S E U}$ is the recorded upset count, $N_{\text {impacts }}$ is the total number of particle strikes, and $A_{\text {irrad }}$ is the area of the irradiated surface which must define a volume enclosing all sensitive volumes (in TIARA we use either a bounding sphere or a bounding box). The $4 \pi$ factor accounts for the total solid angle, and the $A_{\text {irrad }} / 4$ term arises as the average projected area of the irradiation surface when viewed from all angles (as long as it is convex). On a computational note, let us mention that some acceleration is needed for the simulations to run within a reasonable time: for sufficient statistics to be gathered, a few million impacts have to be simulated for each environment, which even with parallelization requires too much computing power. To avoid running all impacts entirely, we discard the calculations if the LET is below a certain cut-off, calculated to be the absolute minimum for an SEU to occur (by considering the longest chord in our sensitive volumes, the maximum bipolar amplification, the minimum critical charge...). For all environments, a huge portion of the LET spectrum stands below this cut-off, which provides a speed-up factor of about 100, resulting in simulation times of about a few hours for one environment.

Direct comparisons between the IRPP upset rates computed in section IV and those calculated with TIARA are given in Fig. 9 (showing error bars from TIARA was deemed unnecessary here since all simulations gathered more than 400 SEU's, with $95 \%$ confidence intervals of $\pm 10 \%=2 / \sqrt{400})$. The IRPP model provides satisfactory estimates of the error rates when TIARA is taken as a reference: overall it correlates with about $2 \times$ precision to our custom response model, with a mismatch depending on the environment LET content, as was to be expected from the LET-dependent modeling of bipolar amplification. Although both methods are in perfect agreement at normal incidence by construct, the notable difference is that with the IRPP model the gradual turn-on of the sensitive volume is forced via a statistical superposition of threshold LET's, when in our specific response model it is explained from the physical insight gained with TCAD. The legend of Fig. 9 aims at depicting this ground difference, showing nested sensitive volumes for TIARA and stacked RPP's for IRPP (even though both models simulate a continuum of those volumes in fact). Nevertheless, for practical purposes this study shows that first-order estimations of the upset rates can be obtained with the IRPP algorithm when taking the silicon film thickness as IRPP $z$ depth. This is mainly due to the fact that parasitic bipolar amplification remains modest at all times in FD-SOI; in Partially-Depleted SOI technologies often featuring $\beta$ larger than 10 [13], this conclusion would probably not hold as the effective collection depth could be much larger than the physical silicon thickness above the BOX.

Taking advantage of the predictive power of the circuit solver coupled to our radiation response model, we can perform further analyses with TIARA: in Fig. 10 we show the effect of voltage scaling on the technology radiation response for different orbits. The simulations show that the upset rate can increase twofold when lowering the voltage by $20 \%$. The reason for this was mentioned earlier: while the critical charge scales linearly with voltage, the collected charge does not vary much with voltage in depleted technologies, and thus the balance between them can shift significantly. Note that the 
effect is symmetrical: strong reductions in SEU rate can also be achieved by raising the supply voltage, providing interesting opportunities for hardened designs. Fig. 10 also points out that the voltage scaling effects are more pronounced in low-LET environments where many ions are close to threshold LET. As an indication, the simulated upset rate for FD-SOI in GEO at solar minimum is below $5 \mathrm{SEU} / \mathrm{Gb} /$ day at all $V_{d d}$, about 50 times lower than bulk simulated with IRPP.

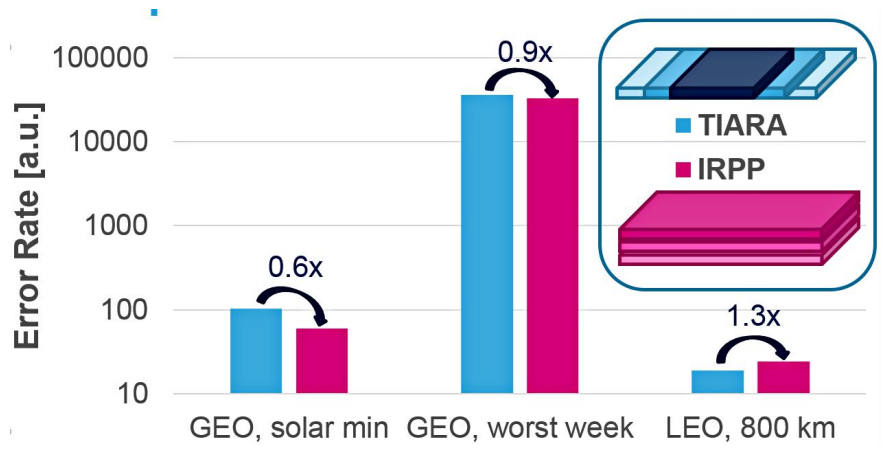

Fig. 9: Comparison of TIARA and IRPP upset rate predictions under different environments: the generic IRPP approach can correlate to our custom response model with $2 \times$ accuracy.

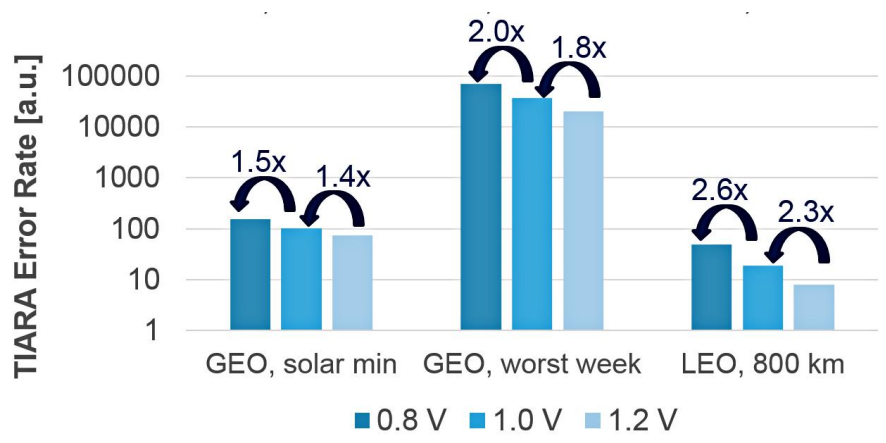

Fig. 10: Investigation of voltage scaling effects with TIARA: the upset rate can increase by $2 \times$ upon $-20 \%$ reduction in supply voltage.

\section{CONCLUSION}

In this study we have discussed methodologies for computing on-orbit Single-Event Upset (SEU) rates for a $28 \mathrm{~nm}$ FDSOI technology. Heavy-ion cross sections of a representative SRAM cell were reported to be two decades lower than an equivalent cell in planar bulk technology. Using simulations of several on-orbit radiation environments, these test data were used to compute upset rates with the Integral Rectangular ParallelePiped (IRPP) model available in CREME, once again highlighting fundamental differences with bulk technology: for reduced sensitive volumes in technologies such as FDSOI or FinFET, confinement deeply impacts the modeling choices and assumptions to be made regarding the cell's radiation response at an angle, given measurements at normal incidence: on our FD-SOI example the upset rate was shown to increase by $30 \times$ when varying the sensitive thickness in the IRPP model from the CREME default setting for bulk to the physical film thickness. For practical usage of the IRPP model in CREME, this study has shown that in $28 \mathrm{~nm}$ FD-SOI using the silicon film thickness as sensitive depth is a good choice, since bipolar amplification is limited in the technology. More accurate upset rates were then computed within the TIARA Monte-Carlo tool chain via a new response model tailored to FD-SOI, after TCAD investigations and calibration against heavy-ion measurements. The SEU rates calculated with IRPP were shown to be in good $(2 \times)$ correlation with the reference TIARA simulations. Additionally, the effects of voltage scaling were investigated with TIARA, revealing $2 \times$ variations in upset rate when moving $20 \%$ away from nominal voltage, as a very consequence of the technology being fully depleted. For geosynchronous earth orbits under quiet Sun conditions, the SEU rate in $28 \mathrm{~nm}$ FD-SOI was found to be of only a few SEU per Gb per day (about $50 \times$ better than in bulk), demonstrating the technology's suitability for space applications.

\section{REFERENCES}

[1] E. Petersen, J. Pickel, J. Adams, and E. Smith, "Rate prediction for single event effects-a critique," Transactions on Nuclear Science, vol. 39, no. 6, pp. 1577-1599, Dec 1992.

[2] [Online]. Available: https://creme.isde.vanderbilt.edu

[3] [Online]. Available: https://www.spenvis.oma.be

[4] P. Roche and G. Gasiot, "Advanced CMOS Bulk, FinFET and UTBB SOI Technologies," in 2014 IEEE Nuclear and Space Radiation Effects Conference, July 2014, section III Short Course Notebook.

[5] A. Virtanen, "Radiation effects facility RADEF," in 2002 Proceedings of the Eighth IEEE International On-Line Testing Workshop, 2002, p. 188.

[6] P. Roche, J.-L. Autran, G. Gasiot, and D. Munteanu, "Technology downscaling worsening radiation effects in bulk: SOI to the rescue," in 2013 IEEE International Electron Devices Meeting (IEDM), Dec 2013 , pp. 31.1.1-31.1.4.

[7] G. Gasiot, D. Soussan, M. Glorieux, C. Bottoni, and P. Roche, "SER/SEL performances of SRAMs in UTBB FDSOI28 and comparisons with PDSOI and BULK counterparts," in 2014 IEEE International Reliability Physics Symposium, June 2014, pp. SE.6.1-SE.6.5.

[8] V. Malherbe, G. Gasiot, D. Soussan, A. Patris, J.-L. Autran, and P. Roche, "Alpha soft error rate of FDSOI 28 nm SRAMs: Experimental testing and simulation analysis," in 2015 IEEE Internationl Reliability Physics Symposium (IRPS), April 2015, pp. SE.11.1-SE.11.6.

[9] A. Tylka, J. Adams, P. Boberg, B. Brownstein, W. Dietrich, E. Flueckiger, E. Petersen, M. Shea, D. Smart, and E. Smith, "CREME96: A Revision of the Cosmic Ray Effects on Micro-Electronics Code," IEEE Transactions on Nuclear Science, vol. 44, no. 6, pp. 2150-2160, Dec 1997.

[10] R. A. Reed, R. A. Weller, M. H. Mendenhall, D. M. Fleetwood, K. M Warren, B. D. Sierawski, M. P. King, R. D. Schrimpf, and E. C. Auden, "Physical Processes and Applications of the Monte Carlo Radiative Energy Deposition (MRED) Code," IEEE Transactions on Nuclear Science, vol. 62, no. 4, pp. 1441-1461, Aug 2015.

[11] P. Roche, G. Gasiot, J. Autran, D. Munteanu, R. Reed, and R. Weller, "Application of the TIARA Radiation Transport Tool to Single Event Effects Simulation," IEEE Transactions on Nuclear Science, vol. 61, no. 3, pp. 1498-1500, June 2014.

[12] K. Castellani-Coulie, D. Munteanu, V. Ferlet-Cavrois, and J. Autran, "Simulation analysis of the bipolar amplification in fully-depleted SOI technologies under heavy-ion irradiations," IEEE Transactions on $\mathrm{Nu}$ clear Science, vol. 52, no. 5, pp. 1474-1479, Oct 2005.

[13] V. Ferlet-Cavrois, C. Marcandella, G. Giraud, G. Gasiot, I. Colladant, O. Musseau, C. Fenouillet, and J. du Port de Poncharra, "Characterization of the parasitic bipolar amplification in SOI technologies submitted to transient irradiation," IEEE Transactions on Nuclear Science, vol. 49 no. 3, pp. 1456-1461, Jun 2002. 